\title{
Extracting gluino endpoints with event topology patterns
}

\author{
Niklas Pietsch, ${ }^{a}$ Jürgen Reuter, $^{b}$ Kazuki Sakurai $^{b}$ and Daniel Wiesler ${ }^{b}$ \\ ${ }^{a}$ University of Hamburg, \\ Luruper Chaussee 149, D-22761 Hamburg, Germany \\ ${ }^{b}$ DESY Theory Group, \\ Notkestr. 85, D-22603 Hamburg, Germany \\ E-mail: niklas.pietsch@desy.de, juergen.reuter@desy.de, \\ kazuki.sakurai@desy.de, daniel.wiesler@desy.de
}

Abstract: In this paper we study the gluino dijet mass edge measurement at the LHC in a realistic situation including both SUSY and combinatorial backgrounds together with effects of initial and final state radiation as well as a finite detector resolution. Three benchmark scenarios are examined in which the dominant SUSY production process and also the decay modes are different. Several new kinematical variables are proposed to minimize the impact of SUSY and combinatorial backgrounds in the measurement. By selecting events with a particular number of jets and leptons, we attempt to measure two distinct gluino dijet mass edges originating from wino $\tilde{g} \rightarrow j j \tilde{W}$ and bino $\tilde{g} \rightarrow j j \tilde{B}$ decay modes, separately. We determine the endpoints of distributions of proposed and existing variables and show that those two edges can be disentangled and measured within good accuracy, irrespective of the presence of ISR, FSR, and detector effects.

KeYWORDS: Supersymmetry Phenomenology

ARXIV EPRINT: 1206.2146 


\section{Contents}

1 Introduction $\quad 1$

2 Benchmark scenarios and simulation setup 4

3 Selection criteria from event topologies 5

4 Kinematic variables for endpoint extraction $\quad 8$

5 Disentangling gluino endpoints $\quad 10$

$\begin{array}{lll}5.1 \text { Scenario A } & 11\end{array}$

$\begin{array}{llr}5.2 & \text { Scenario B } & 12\end{array}$

$\begin{array}{lll}5.3 & \text { Scenario C } & 13\end{array}$

6 Endpoint determination $\quad 14$

$\begin{array}{lll}7 & \text { Conclusions } & 17\end{array}$

\section{Introduction}

The Large Hadron Collider (LHC) has entered an exciting era by seeing a tantalizing excess of Higgs-like events in the mass region around $125 \mathrm{GeV}$. The Higgs boson mass parameter receives a large quantum correction of the order of a cut off scale and hence new physics that stabilizes the weak scale is anticipated to be seen in LHC events.

Supersymmetry (SUSY) is one of the most promising candidates of such new physics models. The Minimal Supersymmetric extension of the Standard Model (MSSM) allows an exact unification of all three forces in the Standard Model (SM), indicating a grand unified theory at a very high energy scale. The lightest SUSY particle (LSP) is stable because of a discrete symmetry in the MSSM, called R-parity, and can be a viable dark matter candidate if it is neutral. The R-parity also makes SUSY events in a collider distinct from the SM background. It implies SUSY particles to be produced in pairs, with each decaying into the LSP through a cascade decay chain, leading to multiple jets, leptons and large missing energy. The LHC experimental collaborations so far have put great effort into searching for a sign of Supersymmetry at the LHC. If Supersymmetry is discovered, the next important task is measuring the properties of SUSY particles. SUSY events contain two LSPs in the final states, which escape detection. In hadron colliders, the only information we can deduce on the LSP momenta is a vector sum of their transverse momenta, on the basis of the assumption that there are no extra missing particles, such as neutrinos, in the event. This makes any measurement about SUSY particles non-trivial and challenging. 
Fortunately, many ideas have already been put forward to address this obstacle (see [1] for a review). The most traditional method is to look for kinematical edges in various invariant mass distributions of the daughter particles [2-7]. The locations of these edges reveal information on the unknown intermediate particle masses in the decay chain. Another approach is to use the family of $M_{T 2}$-based kinematic variables [8-32], which often serves the event-by-event best lower bound on the unknown particle mass of interest. The third option is the polynomial method [33-39], which attempts to determine all the missing momenta in the event by solving the kinematic constraints inherent to the process. This allows to measure all intermediate particle masses simultaneously. Connections among some of those methods have also been studied [31, 32]. Alternatively there exist inclusive methods $[40,41]$ hinting towards the overall mass scale. ${ }^{1}$

However, there are other obstacles in translating those methods into realistic applications. The aforementioned methods, except for the inclusive $M_{T 2}$ version [20-22] and $[40,41]$, to some extent rely on the assumption of a detailed knowledge of the particular SUSY event (e.g. the specific production and decays). But in contrast, SUSY events are generally far from unique and rather possess a large variety of production and decay processes. Since most SUSY events lead to similar final states with multiple jets and missing energy, identification of production and decay is very difficult in a hadron collider environment. $^{2}$ The contamination from SUSY events that we are not interested in is referred to as "SUSY background".

In general, the mass determination methods also require the knowledge on the origin of observed particles: which particle originates from which decay vertex in the cascade decay chains. How much knowledge is required depends on the corresponding method. For the edge method, the assignments of particles which do not involve the invariant mass of interest are irrelevant. For the inclusive $M_{T 2}$ method, only the division of particles into two groups matters, but the permutation inside each group is irrelevant. For the polynomial method, the perfect particle assignment is required. ${ }^{3}$ In SUSY events, gluinos and squarks promptly decay into multiple jets, leptons and the LSP. There is a large combinatorial number of particle assignments in the final state to the decay chains, but any such information on the assignment is not accessible in the detector. The wrong assignments, called "combinatorial background", are thus in general irreducible. Both the SUSY background and the combinatorial background often cause a serious impact on SUSY mass measurements.

Recently, several ideas to handle the combinatorial background have been proposed [ $45-$ 48]. Several studies [46-48] suggest that the kinematical edge method can effectively reduce the combinatorial background in the context of the $M_{T 2}$-based method in the $\tilde{g} \tilde{g} \rightarrow 4 j+2 \tilde{\chi}_{1}^{0}$ process. In this method, the dijet invariant mass edge for the $\tilde{g} \rightarrow j j \tilde{\chi}_{1}^{0}$ decay is assumed to be already known. The position of this edge is then used as follows: any assignment

\footnotetext{
${ }^{1}$ Recently, there was a new study [42] which as well utilizes event topologies and invariant mass shapes for mass determination.

${ }^{2}$ For an interesting study along this line, see ref. [43].

${ }^{3}$ Some permutations in the same decay chain may be irrelevant if there is a mass hierarchy between initially produced particles and the LSP [44].
} 
having the jet pairs exceed this gluino dijet mass edge is assumed to be a wrong one and rejected. Although this method offers a good performance, the measurement (identification and position) of the gluino dijet mass edge itself suffers from SUSY and combinatorial backgrounds and deserves a careful study.

The aim of this paper is to assess the feasibility of the gluino dijet mass edge measurement in a realistic collider study including SUSY background, the effect of initial state radiation (ISR), and a finite detector resolution. Compared to resonance peaks, endpoints of distributions are formed by very few events and are thus very vulnerable to backgrounds and momentum mismeasurements.

The relevance of ISR on the gluino mass measurement has recently been pointed out $[49,50]$ (for an early study on the effect of ISR on jet measurements in LHC events, cf. [51]). Events with a high $p_{T}$ ISR jet intermingling with decay jets appear much more frequently within the $\tilde{g} \tilde{g}$ than in the $\tilde{q} \tilde{q}$ production process.

In addition to ISR, we would like to emphasize the importance of the SUSY background in the measurements. If squarks are kinematically accessible at the LHC, the $\tilde{q} \tilde{g}$ associated production has in general larger cross sections than the $\tilde{g} \tilde{g}$ production. The subsequent decay of the $\tilde{q}$ increases the number of unwanted jets and also may decrease the number of signal gluinos in the event if it decays to a wino or bino state directly. Moreover, if the wino states lie in between the gluino and the LSP masses, gluino and left-handed squarks decay in general more frequently into the wino states first, followed by the subsequent wino decay into the LSP: $\tilde{g} \rightarrow j j \tilde{\chi}_{i} \rightarrow j j j j \tilde{\chi}_{1}^{0}$ or $\tilde{q}_{L} \rightarrow j \tilde{\chi}_{i} \rightarrow j j \tilde{\chi}_{1}^{0}$, where $\tilde{\chi}_{i}$ is either $\tilde{\chi}_{1}^{ \pm}$or $\tilde{\chi}_{2}^{0}$. Those processes do have a significant impact on the gluino dijet mass edge measurement. The location of the edge in the $\tilde{g} \rightarrow j j \tilde{\chi}_{i}$ events, from hereon entitled as the "wino edge", is smaller than that in the $\tilde{g} \rightarrow j j \tilde{\chi}_{1}^{0}$ events, which we choose to name "bino edge".

Therefore, in the inclusive sample, the structure of the bino edge is weakened because of the overwhelming $\tilde{g} \rightarrow j j \tilde{\chi}_{i}$ events, and the contribution from $\tilde{g} \rightarrow j j \tilde{\chi}_{1}^{0}$ events overshoots the wino edge. This last point is particularly problematic, since this overshooting is able to mimic disturbing effects of hadronization and detector response, even when two jets from the same decay chain and gluino jets are unambiguously selected. In this study we attempt to disentangle those two edges by selecting events with a particular range for the multiplicity of jets and leptons.

Note that both the presence of high- $p_{T}$ ISR jets and jets from irreducible SUSY background contributes to the combinatorial background. For instance in a $\tilde{g} \tilde{g} \rightarrow 4 j+\tilde{\chi}_{1}^{0}$ event, the ratio between correct and wrong jet pairs is $1 / 3$. On the other hand, in the $\tilde{g} \tilde{q}+j_{\text {ISR }} \rightarrow 4 j+\tilde{\chi}_{i} \tilde{\chi}_{1}^{0} \rightarrow 6 j+2 \tilde{\chi}_{1}^{0}$ events with two of those jets failing to satisfy a jet identification criteria (leading to the same $4 j+$ missing energy event), it is either $1 / 6$ or 0 depending on whether or not one of the gluino jets is lost.

In order to make the problem tractable and the study as generic as possible, in this paper we use a (semi-)simplified model, where all the higgsinos, sleptons and the third generation squarks are decoupled and an approximate grand unified theory (GUT) relation 6:2:1 is imposed on the three gaugino masses. We also concentrate on the situation where the squarks are heavier than the gluino for the following reasons: This type of mass spectra is motivated by an observed excess of Higgs-like events in the mass range around $125 \mathrm{GeV}$, 
since such a relatively heavy Higgs boson mass indicates possibly heavy third generation squarks in the MSSM. In any case, the mass ordering between the gluino and squarks (if they are both present at the LHC) are likely to be observed by looking at the distributions of the hemisphere mass or the $M_{T 2}$ in the high $p_{T}$ dijet events [50]. Although the semisimplified model has much fewer parameters than the MSSM, it can nevertheless cover the dominant features in most of the interesting SUSY spectra which appear in popular SUSY-breaking scenarios such as gravity and gauge mediation.

The paper is organised as follows. In section 2 we introduce our three benchmark scenarios under investigation covering the different kinematic and phenomenological features, as well as our event simulation setup. In section 3, we discuss the topological event configurations arising in our study and introduce a method of endpoint selection by means of semi-inclusive jet multiplicities. Then, we propose new variables and compare them to existing ones in section 4, give numerical results in section 5 and estimate the actual endpoint positions in section 6 before concluding in section 7 .

\section{Benchmark scenarios and simulation setup}

Since our interest is the gluino three body decay, $\tilde{g} \rightarrow j j \tilde{B}(\tilde{W})$, we focus on scenarios with squarks heavier than the gluino (otherwise, $\tilde{g} \rightarrow j \tilde{q}$ dominates the gluino branching ratios). For the study of the impact of SUSY backgrounds, we introduce a semi-simplified model in order to keep the problem parametrically manageable and the discussion as general as possible. The higgsino states are decoupled, therefore the lightest neutralino is a pure composed bino state, and similarly the second lightest neutralino and the lighter chargino are purely composed of the wino states. We adopt an approximate GUT relation of 6:2:1 of the three gaugino masses and fix them to $\left(m_{\tilde{g}}, m_{\tilde{W}}, m_{\tilde{B}}\right)=(1200,400,200) \mathrm{GeV}$. The gluino dijet mass edges are then given by $m_{j j}^{\max }=m_{\tilde{g}}-m_{\tilde{B}}=1000 \mathrm{GeV}$ for the $\tilde{g} \rightarrow j j \tilde{B}$ process and $m_{j j}^{\max }=m_{\tilde{g}}-m_{\tilde{W}}=800 \mathrm{GeV}$ for the $\tilde{g} \rightarrow j j \tilde{W}$ process. Sleptons and third generation squarks we explicitly decouple, since their presence may in any case help disentangle the combinatorial issues of the underlying SUSY cascade using leptons and b-tagging (or in the worst case not deteriorate the method presented here). The first two generation squarks are assumed to be degenerate and define the following three scenarios (see also table 1):

Scenario A $\left(\boldsymbol{m}_{\tilde{q}}=\mathbf{1 3 0 0} \mathrm{GeV}\right)$. The associated $\tilde{q} \tilde{g}$ production dominates the SUSY production processes. $\Delta m \equiv m_{\tilde{q}}-m_{\tilde{g}}=100 \mathrm{GeV}$ and the branching fraction of $\tilde{q} \rightarrow j \tilde{g}$ is kinematically suppressed. The squarks do mainly decay to the lighter gauginos, $\tilde{q} \rightarrow j \tilde{B}(\tilde{W})$, and we expect a prominently hard jet coming from the squark decay in addition to only one signal gluino in the dominant combined production/decay process.

Scenario B $\left(m_{\tilde{q}}=1900 \mathrm{GeV}\right)$. The squark production starts getting suppressed because of the heavier mass, but the associated $\tilde{q} \tilde{g}$ production still has a sizable cross section. The mass splitting between squarks and gluino is relatively large: $\Delta m \equiv$ $m_{\tilde{q}}-m_{\tilde{g}}=700 \mathrm{GeV}$. The main decay mode of the squarks is $\tilde{q} \rightarrow j \tilde{g}$. We expect a moderately hard jet coming from the squark decay as well as two signal gluinos in the dominant combined production/decay process. 


\begin{tabular}{|c|c|c|c|c|c|c|}
\hline spectrum & $m_{\tilde{Q}}$ & $m_{\tilde{G}}$ & $m_{\tilde{W}}$ & $m_{\tilde{B}}$ & $m_{j j}^{\max }(\tilde{W})$ & $m_{j j}^{\max }(\tilde{B})$ \\
\hline $\mathrm{A}$ & 1300 & 1200 & 400 & 200 & 800 & 1000 \\
$\mathrm{~B}$ & 1900 & 1200 & 400 & 200 & 800 & 1000 \\
$\mathrm{C}$ & 10000 & 1200 & 400 & 200 & 800 & 1000 \\
\hline
\end{tabular}

Table 1. Relevant sparticle masses in $\mathrm{GeV}$ for the three benchmark spectra under investigation. All other scalars and higgsinos were set to $10 \mathrm{TeV}$.

Scenario $\mathbf{C}\left(\boldsymbol{m}_{\tilde{q}}=\mathbf{1 0 0 0 0} \mathrm{GeV}\right)$. The squarks are decoupled and not produced at the LHC. The $\tilde{g} \tilde{g}$ process is the unique SUSY QCD production process.

Throughout this paper, we use the following setup of simulation tool chain: SUSY events were generated using Herwig ++ [53] and WHIZARD [54-56]. Furthermore, the events are inclusive in that they are passed through the full simulation chain, i.e. decay, parton showering, hadronization and detector simulation. The detector responses are simulated by the DELPHES package [58] using CMS detector settings. The anti- $\mathrm{k}_{T}$ algorithm [59-61] with jet resolution parameter $R=0.5$ is adopted, and only jets with $p_{T}>50 \mathrm{GeV}$ and $|\eta|<2.5$ are accepted to suppress the soft activities coming from initial and final state radiation and the underlying event. Based on [62] the following baseline selection cuts are applied to all events:

- $H_{T}>800 \mathrm{GeV}$

- $E_{T}^{\text {miss }}>200 \mathrm{GeV}$

- $\Delta \phi\left(j_{1 / 2}, E_{T}^{\text {miss }}\right)>0.5$

where $H_{T}$ is defined as the scalar sum of the first four hardest jets and $j_{1 / 2}$ denotes the hardest or second hardest jet, respectively. These cuts are designed to suppress SM backgrounds. The cut on $\Delta \phi$ between $E_{T}^{\text {miss }}$ and the hardest and second hardest jet, respectively, is applied to reject events where $E_{T}^{\text {miss }}$ originates from jet energy mismeasurements.

\section{Selection criteria from event topologies}

Many existing studies address the combinatorial issue in a scenario comparable to type $\mathrm{C}$ and assume that the gluino has just a single decay mode: $\tilde{g} \rightarrow j j \tilde{B}$. However, in most of the interesting and relevant SUSY spectra which are suggested by several SUSY breaking scenarios, the gluino has at least two comparable decay modes: $\tilde{g} \rightarrow j j \tilde{B}$ and $\tilde{g} \rightarrow j j \tilde{W}$, each of which has a different dijet mass edge. Since the bino is lighter than the wino, the position of the bino edge is higher than the wino edge and the $\tilde{g} \rightarrow j j \tilde{B}$ process is a serious background for the wino edge measurement. Because of the larger gauge coupling of the wino the gluino decays much more frequently into the wino. Therefore even in the case that the wino edge is smaller than the bino edge, the $\tilde{g} \rightarrow j j \tilde{W}$ gives a significant contribution 
right below the bino edge which makes the bino edge measurement rather difficult. If a gluino directly decays to a wino, the wino subsequently decays as follows:

$$
\begin{aligned}
\tilde{W}^{0} & \rightarrow h+\tilde{B} \rightarrow b b \tilde{B} \\
\tilde{W}^{ \pm} & \rightarrow W^{ \pm}+\tilde{B} \rightarrow j j(l \nu)+\tilde{B}
\end{aligned}
$$

In this study, we do not use b-tagging and treat b-jets as non-tagged jets. As can be seen, the inclusion of the $\tilde{g} \rightarrow j j \tilde{W}$ process not only introduces a confusing extra endpoint but also increases the number of jets in the event leading to a drastic increase of the number of wrong jet pairings. Consequently, it makes it more difficult to choose the correct dijet pair coming from a gluino three body decay. In order to separately measure two gluino endpoints, we extract two sub-samples where one of which mostly contains $\tilde{g} \rightarrow j j \tilde{B}$ and the fraction of $\tilde{g} \rightarrow j j \tilde{W}$ is reasonably suppressed, and vice versa in the other sample. To do so, we focus on the fact that the number of the final state particles increases if the event contains the $\tilde{g} \rightarrow j j \tilde{W}$ process.

In figure 1, we classify the event topology in terms of the number of decay products of SUSY cascade decay chains. As can be seen, if we choose the events having less than or equal to four SUSY decay products, we can unambiguously select $\tilde{g} \rightarrow j j \tilde{B}$. On the other hand, $\tilde{g} \rightarrow j j \tilde{W}$ can be unambiguously selected if we look at the events with more than or equal to eight SUSY decay products.

At detector level in a fully hadronic LHC event, it is clear that we are not able to directly observe the number of SUSY decay products. However, this number should be correlated to the number of observed jets in the detector, even after taking into account the effects of initial and final state radiation, underlying event, hadronization and detector acceptances. In figure 2, we show this correlation between the number of SUSY decay products and the number of observed jets in the detector. Keep in mind, that we only take account of jets satisfying $p_{T}>50 \mathrm{GeV}$ and $|\eta|<2.5$. As can be seen from there, this correspondence is significantly smeared out by all the undesired radiation, hadronization and detector effects, but it is nevertheless still visible. This observation enables us to propose the following two criteria for the separation of the two different edges:

- bino edge: 4-5 jets \& lepton veto,

- wino edge: $\geq 6$ jets \& 1 lepton.

These are the basis of the semi-inclusive jet multiplicity endpoint selection. For the bino selection, we opt for $4-5$ jet bins rather than selecting $\leq 4$ jets events. In the $4-5$ jets events, wino contamination is slightly larger than in the $\leq 4$ jets events. However, the $\leq 4$ jets events generally suffer from rather large Standard Model backgrounds and a small signal cross section (see figure 2). On the other hand, some level of wino contamination is acceptable in the bino edge measurement, because the bino endpoint is larger than the wino endpoint, and the wino contamination is naturally suppressed at the vicinity of the bino endpoint. For the wino selection, instead of taking a $\geq 8$ jets sample we select $\geq 6$ jets +1 lepton. By lepton we mean either an electron or a muon with $p_{T}>20 \mathrm{GeV}$ and $|\eta|<2.5$. This is due to a leptonically decaying $W$, with which we are able to decrease the 


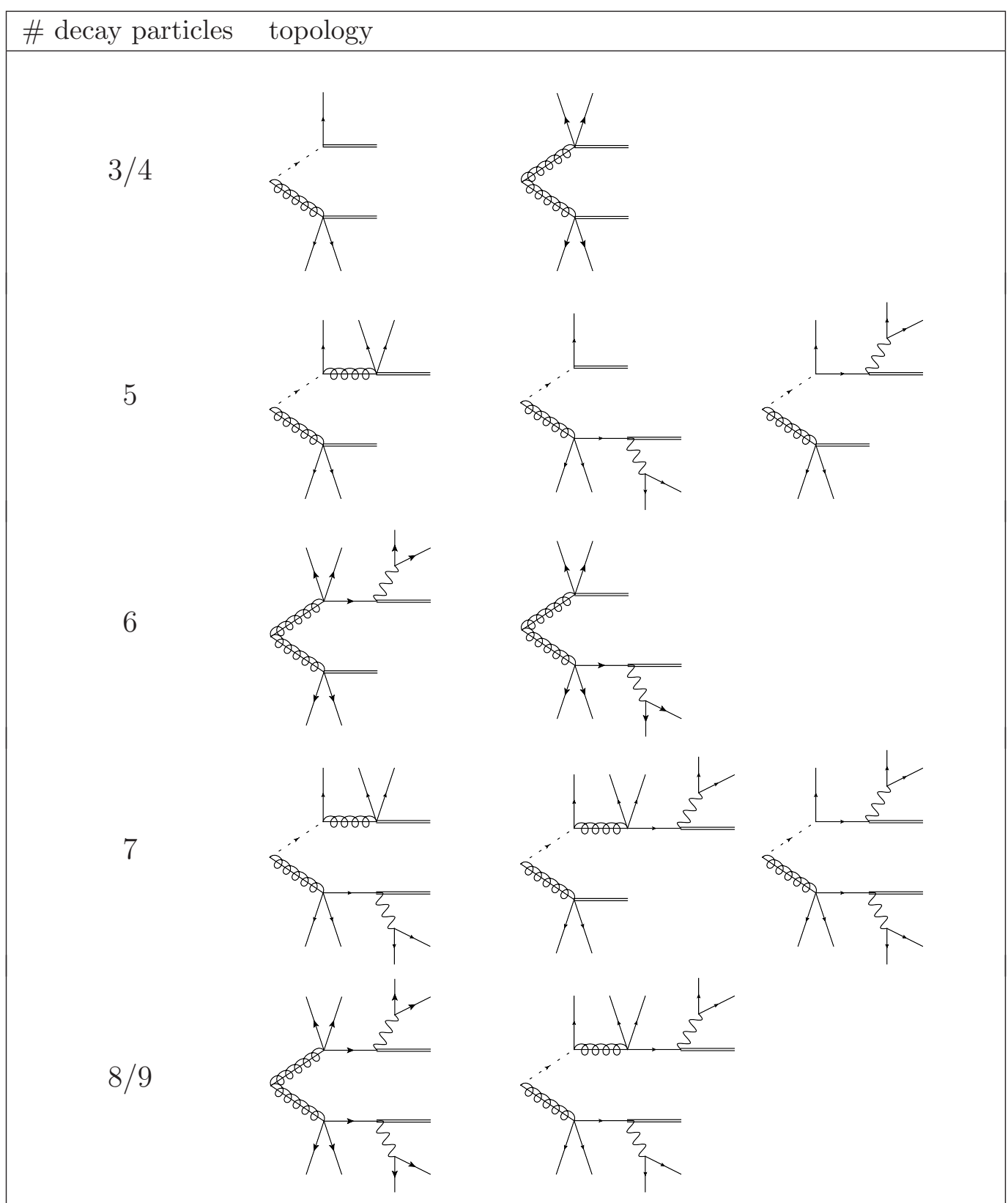

Figure 1. Topological decay configurations of dominant gluino signals, sorted by number of decay particles, represented by solid lines in the final state. Double lines denote the invisible LSP.

overall number of jets in the event by two (see eq. (3.2)) and thus drastically reduce the number of wrong jet parings.

However, we should keep in mind that the correlation shown in figure 2 is not too strong. The restriction to a maximum of four or five jets thus kills a lot of the wino contamination, but the ratio $N(\tilde{B}) / N(\tilde{W})$ of bino to wino events still remains in the ballpark of $0.3-0.7$, depending upon the spectrum. Extending the number of jets up to five is indispensable for types $\mathrm{B}$ and $\mathrm{C}$ since it increases the statistics at the price of a slightly 



Figure 2. Inclusive number of jets vs expected number of partonic colored particles from topological considerations. In the left, center and right plot scenarios of type $\mathrm{A}, \mathrm{B}$ and $\mathrm{C}$ are shown. Cuts for detector level jets are $p_{T}>50 \mathrm{GeV}$ and $|\eta|<2.5$.

reduced sample purity, i.e. ratio of bino to wino events. The relative ratio of wino to bino events in the wino selection is a lot better than in the bino case: $N(\tilde{W}) / N(\tilde{B}) \sim \mathcal{O}(10)$.

After defining the two relevant selection criteria, we are able to discuss the corresponding SM background contributions in more detail. The dominant processes after the bino endpoint selection are QCD multijet production, where $E_{T}^{\text {miss }}$ originates from neutrinos produced in heavy flavor quark decays and jet energy mismeasurements due to instrumental effects, and the production of neutrino pairs from $Z$ boson decays in association with hard jets $(Z+$ jets $)$. The production of leptonically decaying $t \bar{t}$ pairs and leptonically decaying $W$ bosons in association with hard jets $(W+$ jets $)$ is expected to be suppressed by the lepton veto. To further reduce the background a cut on $Y_{M E T}=E_{T}^{\text {miss }} / \sqrt{H_{T}}$, which is used in SUSY searches at ATLAS and CMS [63, 64], can be introduced in addition.

In contrast to the bino selection the wino endpoint selection criteria are expected to suppress the SM background in a way that allows to extract endpoints without applying further cuts. By requiring one lepton on top of the baseline selection mentioned above the background from QCD multijet production is anticipated to be completely suppressed. By selecting events with at least six hard jets most of $W+$ jets and $Z+$ jets events are also expected to be rejected.

\section{Kinematic variables for endpoint extraction}

Our semi-inclusive jet samples contain 4 or 5 jets for the bino selection and $\geq 6$ for the wino selection, and we should therefore determine how to select gluino dijets out of a large number of possible jet pairings. In the event samples there are many jets that are not originating from the gluino three body decay. Those are mainly coming from ISR as well as from the $W$ decay. In the scenarios $\mathrm{A}$ and $\mathrm{B}$, an additional jet comes from the squark decay, too. It can be argued that the gluino dijets have relatively high $p_{T}$ compared to the ISR and $W$ decay products, which as a kinematical effect is easy to understand.

Figure 3 shows the abundance of gluino, squark and wino jets in $i$-th hardest jet, sorted by $p_{T}$, for the three benchmark scenarios. The identification is done using MC truth by matching detector level jets to partonic objects. Objects, which are not successfully assigned to originate from SUSY mothers are thus either from ISR or other clustering 



Figure 3. Abundances of gluino (black full line), squark (red dashed line), wino (blue full line) and ISR/unknown jets (blue dotted line) are shown in an inclusive sample for jet bins of hardness $i$ in scenarios $\mathrm{A}, \mathrm{B}$ and $\mathrm{C}$ from left to right.

effects of the jet algorithm. As can be seen, for scenarios A, B and C, the first three $p_{T}$-hardest jets are most likely coming from the gluino decay. However for scenarios A and $\mathrm{B}$, there is a significant contribution in the highest $p_{T}$ jet bin from the squark decay $\tilde{q} \rightarrow j \tilde{B}(\tilde{W})$. If the squark jet is wrongly selected and contributes to the distribution, there is a high risk to exceed the correct gluino endpoint because the dijet mass tends to be large because of the large $p_{T}$ of the squark jet. This is the motivation for the minimization procedure introduced in the following variables, which takes care of this:

$$
\begin{aligned}
\min _{3 j} & =\min _{k=1,2}\left\{m_{3 k}\right\} \\
\min _{123} & =\min _{i, j=1,2,3}\left\{m_{i j}\right\}, \quad i \neq j
\end{aligned}
$$

Here $m_{i j}$ denotes the invariant mass of jets $i$ and $j$. The endpoint of $\min _{3 j}$ is expected to be the same as the gluino endpoint as long as one of the first two highest $p_{T}$ jets and the third highest $p_{T}$ jet are coming from the same gluino decay. The $\min _{123}$ is smaller than $\min _{3 j}$ event-by-event because of the wider range of the minimization and has the same endpoints as the gluino's as long as two of the first three highest $p_{T}$ jets are coming from the same gluino decay. In scenario A, the relative abundance of gluino jets does not look very promising. However keeping in mind, that most of the time we are left with only one gluino, the two variables proposed above we expect to also work reasonably well.

In scenario $\mathrm{B}$, it is reasonable to explicitly exclude the highest $p_{T}$ jet bin and build a distribution out of the remaining three hardest jets, since the gluino jets have the highest relative abundance in these bins. This is the motivation behind the following variable:

$$
\min _{234}=\min _{i, j=2,3,4}\left\{m_{i j}\right\}, \quad i \neq j
$$

Here we explicitly remove the highest $p_{T}$ jet and select the dijet pair among the second, third and fourth highest jets, which yields the smallest invariant mass. This variable should have the same endpoint as the gluino if two of those jets are being originated from the same gluino decay.

There exist other methods in the literature, which address parts of this particular problem of combinatorics in gluino endpoint extraction. Two prominent examples are 
the hemisphere method [52] as well as a topological method for 4 jets $+\mathbb{E}_{T}$ proposed in [43]. We give a brief overview over both these methods as we compare them to our kinematical variables.

In the hemisphere method, every event is divided into two hemispheres defined by two seeds. These are usually taken to be the hardest object in the event and the one that maximizes the variable $\Delta R \cdot p$, with $\Delta R=\sqrt{\Delta \eta^{2}+\Delta \phi^{2}}$. Then all objects are subsequently clustered to one of the two spatial areas, defined by the seeds. This is done by assigning each particle to that hemisphere which minimizes the value of the Lund distance measure,

$$
d\left(p_{k}, p_{j}^{(s)}\right)=\left(E_{j}-p_{j}^{(s)} \cos \theta_{j k}\right) \frac{E_{j}}{\left(E_{j}+E_{k}\right)^{2}}
$$

between the four momentum $p_{k}$ of the object to be associated and the two seed momenta $p_{1}^{(s)}$ and $p_{2}^{(s)}$. After all objects are clustered, the seeds are updated to be the sum of all objects in the corresponding hemisphere. Finally, the procedure is iterated until the assignment converges (more details on the specifics of this algorithm can be found in [52]). Once two hemispheres are obtained, we pick up the first two highest $p_{T}$ jets from one of the two hemispheres, which defines the following two variables:

$$
m_{12}^{(1)}=m_{12} \quad(\text { from hemisphere } 1), \quad m_{12}^{(2)}=m_{12} \quad(\text { from hemisphere } 2),
$$

where hemisphere 1 is defined as the hemisphere which contains the highest $p_{T}$ jet in the event.

Concerning the second method, in ref. [43], the authors have studied the possibility of identifying the dominant event topology in exclusive 4 jet $+\mathbb{E}_{T}$ events. For this purpose, they defined two dijet mass variables, $F_{3}$ and $F_{4} . F_{3}$ is designed for event topologies where 3 jets come from the same cascade chain and 1 jet from the other one. It is given by

$$
F_{3}\left(p_{1}, p_{2}, p_{3}, p_{4}\right)=m_{k l}, \quad \text { for } \quad \epsilon_{i j k l} \neq 0 \quad \text { and } \quad m_{i j}=\max _{r, s=1, \ldots, 4}\left\{m_{r s}\right\}
$$

which is the invariant dijet mass opposite to the maximum of all possible dijet masses. This variable has the same endpoint as the largest dijet mass endpoint originating from the cascade chain producing the 3 jets. $F_{4}$ is, in contrast, designed for the symmetric event topology where both the cascade chains produce 2 jets each. The definition of this variable is given by

$$
F_{4}\left(p_{1}, p_{2}, p_{3}, p_{4}\right)=\min _{i, j=1, \ldots, 4}\left\{\max \left(m_{i j}, m_{k l}\right)\right\}, \quad \epsilon_{i j k l} \neq 0 .
$$

which is the minimum of the larger dijet mass pair out of three possible combinations. It has the same endpoint as the dijets coming from the same cascade chain each. Although those variables have originally been defined to address exclusive 4 jet $+\mathbb{E}_{T}$ events, we use them for our bino and wino selection samples by applying them to just the first four highest $p_{T}$ jets.

\section{$5 \quad$ Disentangling gluino endpoints}

In this section, we show the distributions of the variables defined in the previous section. We assume $\mathrm{LHC}$ at $14 \mathrm{TeV}$ with an integrated luminosity of $300 \mathrm{fb}^{-1}$, which corresponds to 

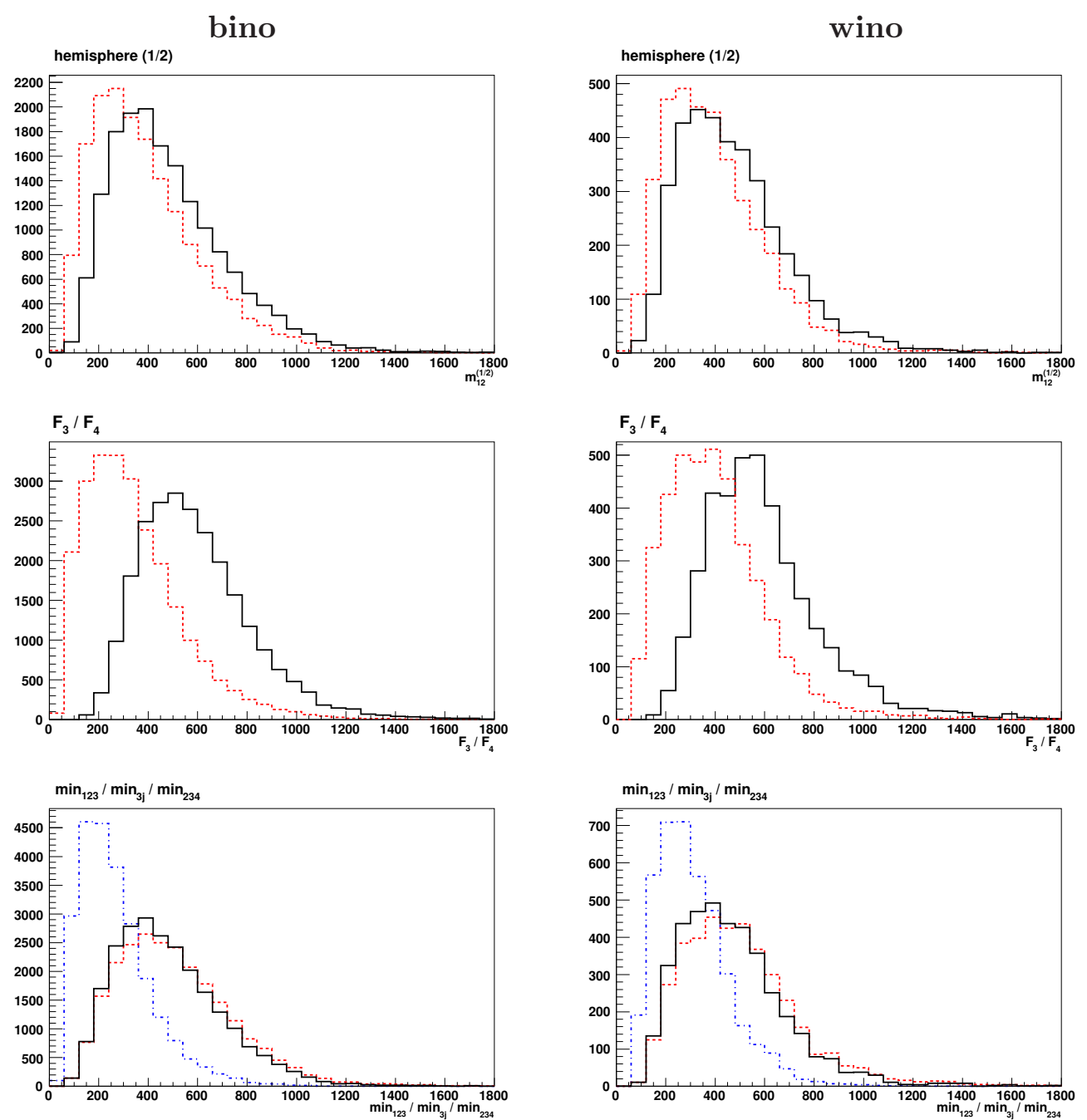

Figure 4. Scenario A: first row: invariant mass of two hardest objects $m_{12}^{(1)}$ and $m_{12}^{(2)}$ for hemispheres 1 (solid, black) and 2 (dashed,red). Second row: F4 (solid, black) and F3 (dashed,red). Third Row: $\min _{123}$ (solid, black), $\min _{3 j}$ (dashed,red) and $\min _{234}$ (dot-dashed, blue). All variables are defined in section 4 . The left and right column correspond to the bino and wino selection criteria, respectively.

108k, 27.6k, and 16.2k signal events for scenarios of type A, B and C, respectively, according to the leading order cross section computed by Herwig ++ . Notice, that these numbers are conservative in that SUSY QCD NLO corrections for squark and gluino production are known to increase cross sections by a K-factor of up to 2 [57]. In the simulation we take account of all the QCD productions, possible decays, and the effects of parton showering, hadronization and detector simulation.

\subsection{Scenario A}

In scenario A, the mass splitting between squark and gluino is only $100 \mathrm{GeV}$. The associated $\tilde{q} \tilde{g}$ process dominates the SUSY production. The squarks decay preferably into bino or wino 
directly because the decay mode into gluino is kinematically suppressed. This reduces the number of signal gluinos from two to one compared to the other scenarios. The 'squark jet' coming from $\tilde{q} \rightarrow j \tilde{B}(\tilde{W})$ has a significant $p_{T}$ (c.f. figure 3 ) and it should be avoided in favorable dijet combinations, either by minimization or explicit removal for example in the construction of $\min _{234}$.

Figure 4 shows the distributions of the various variables discussed in the previous section for the scenario A. In the left (right) row, the bino (wino) event selection (4 or 5 jets for bino, $\geq 6$ jets $\& \geq 1$ lepton for wino) is applied. In the two plots on the top, the dijet is chosen as the first two highest $p_{T}$ jets in one of the two hemisphere groups. The black solid histograms consider the hemisphere 1 , which contains the highest $p_{T}$ in the event and the red dashed histograms consider the other hemisphere group (hemisphere 2 ). In the middle line, the black solid histograms show $F_{4}$ and the red dashed represent $F_{3}$. The bottom plots show $\min _{123}$ (black solid), $\min _{3 j}$ (red dashed) and $\min _{234}$ (blue dot-dashed).

For the bino edge measurement, $F_{3}$ and $F_{4}$ fail to recover the correct edges. $\min _{123}$ and $\min _{3 j}$ have very similar distributions, but nonetheless both have a slight tendency to overshoot the correct endpoint. The hemisphere variables $m_{12}^{(1)}$ and $m_{12}^{(2)}$ also have endpoint structures around the true bino edge. Especially $m_{12}^{(2)}$ from the softer hemisphere looks most promising out of the examined variables. This is expected due to typically only one gluino and the asymmetric nature of the signal in scenario A.

For the wino edge measurement, most of the distributions have tails above the correct value. These tails are bigger for $\min _{123}$ and $\min _{3 j}$ and a kink structure is less pronounced. However, both $m_{12}^{(2)}$ and $F_{3}$ show a nice edge structure at the vicinity of the true endpoint.

\subsection{Scenario B}

In the type B spectrum, the squark is marginally heavier than in scenario A and the associated $\tilde{q} \tilde{g}$ production still has a sizable cross section compared to the $\tilde{g} \tilde{g}$ process. The main squark decay mode is $\tilde{q} \rightarrow j \tilde{g}$. As this 'squark jet' has a large $p_{T}$ compared to the other jets (c.f. figure 3), it is quite problematic because it is likely to be in the first three highest $p_{T}$ jets and thus increases the number of wrong jet pairings.

Figure 5 shows the distributions of the variables in scenario B. It is obvious that many of the distributions get shifted to higher mass regions and overshoot the true endpoint. For the bino edge measurement, the $m_{12}^{(1)}$ and $m_{12}^{(2)}$ variables obtained from the hemisphere method work quite well. The $F_{4}$ variable significantly overshoots the true endpoint, while $F_{3}$ seems to recover the gluino endpoint, although the slope around the endpoint is quite shallow. The distributions of $\min _{123}$ and $\min _{3 j}$ are again very similar. They exhibit a long tail above the gluino mass edge but some structure seems still visible at the vicinity of the true endpoint.

For the wino edge measurement, most of the variables fail to recover the correct endpoint. $\min _{234}$ on the other hand has a structure around the true wino edge. A good behaviour for $\min _{234}$ is expected in scenario $\mathrm{B}$, since the highest $p_{T}$ jet bin has a dangerous contamination from the squark jet, and gluino jets are more safely to be picked up from the second, third and fourth highest $p_{T}$ jets (cf. figure 3 or section 4 ). 



Figure 5. Scenario B: performance of the variables under investigation. For details, see description of figure 4 .

\subsection{Scenario C}

In the type $\mathrm{C}$ scenario, decoupled squarks with masses of $10 \mathrm{TeV}$ are not accessible at the LHC energy and the $\tilde{g} \tilde{g}$ production is the unique SUSY QCD production. Many studies regarding combinatorial issues are based on this type of spectrum, and most of them consider only one particular gluino decay mode: $\tilde{g} \rightarrow j j \tilde{B}$.

In figure 6 , we show the results for this spectrum. As can be seen, almost all variables successfully recover the wino edge at $800 \mathrm{GeV}$. For the bino event selection, most variables suffer from poor statistics in the vicinity of the true endpoint, $1000 \mathrm{GeV}$. It seems $F_{4}$, $\min _{123}$ and $\min _{3 j}$ perform quite well since their distribution linearly fall down to the true endpoint. 



Figure 6. Scenario C: performance of the variables under investigation. For details, see description of figure 4 .

\section{Endpoint determination}

We attempt to estimate the endpoints of the distributions quantitatively by adopting the edge-to-bump method proposed in [65]. In that method, "kinky" features of distributions which could be originating from an underlying kinematic feature of the distribution or simply be artifacts of statistical fluctuations as well as trigger and cut effects distorting originally smoother distributions, are turned into bumps. Bumps are far easier accessible to data analysis methods, and it is easier to define a systematic error on the procedure of edge determination as this is translated to a statistical error of pseudo-experiments with different edge-to-bump conversion parameters. Roughly speaking, the edge-to-bump method fits the changes in the slopes of the distributions and translates them into peaks. The more distinct a peak is, the more likely it is that there is a true kinematic kink in the original distribution. In this method, for that purpose a naive linear kink fit is used. The 



Figure 7. Examples of bino and wino selection in scenario B and C, respectively: we use the edge-to-bump method to obtain a naive endpoint distribution of the variables $\min _{3 j}$ (bino, first row) and $\min _{123}$ (wino, second row).

resulting value of the fit usually suffers from a non-negligible correlation to the fitting range set by hand. To minimize this artifact, we carry out 1000 naive kink fits with randomly generated fitting ranges and obtain a statistical distribution of endpoint positions.

Figure 7 shows two examples of endpoint distributions. They are obtained by fitting two different distributions for the bino and wino selection in scenario B and C. As we can see, the peak of the distribution is strongly correlated with the theoretically expected endpoint.

As a first estimate one might give the mean value and the corresponding standard deviation as associated error. However, smaller peaks and non-negligible contributions far off the main peak lead to shifted mean values and considerably large errors $(\mathcal{O}(300 \mathrm{GeV}))$. Thus to quantitatively estimate the actual endpoint position and get rid of the contributions far away from the main peak, we apply the following procedure:

1. calculate the mean value $\hat{m}_{0}$ and standard deviation $\sigma_{0}$ of the complete distribution

2. redefine the range of the distribution according to the above values: $\hat{m}_{0} \pm 2 \sigma_{0}$

3. calculate a new mean value $\hat{m}_{i}$ and standard deviation $\sigma_{i}$ inside the range defined above

4. use $\hat{m}_{i}$ and $\sigma_{i}$ as a new seed and start over with point 2

Iterating three to six times the steps 2-4, we find convergence of $\hat{m}_{i}$ and $\sigma_{i}$. The resulting mean values and errors obtained by this procedure are listed in table 2. A first conclu- 


\begin{tabular}{|c|c|c|c|c|c|c|c|}
\hline endpt. & $\min _{123}$ & $\min _{234}$ & $\min _{3 j}$ & $m_{12}^{(1)}$ & $m_{12}^{(2)}$ & $F_{3}$ & $F 4$ \\
\hline \multicolumn{7}{|c|}{ scenario A } \\
\hline bino & $1106 \pm 52$ & $570 \pm 14$ & $1125 \pm 106$ & $822 \pm 21$ & $\mathbf{1 0 1 2} \pm \mathbf{1 0 4}$ & $686 \pm 33$ & $1191 \pm 132$ \\
wino & $908 \pm 83$ & $665 \pm 34$ & $948 \pm 99$ & $932 \pm 31$ & $\mathbf{7 8 0} \pm \mathbf{2 6}$ & $\mathbf{7 9 4} \pm \mathbf{3 3}$ & $1031 \pm 53$ \\
\hline \multicolumn{7}{|c|}{ scenario B } \\
\hline bino & $\mathbf{9 8 6} \pm \mathbf{3 6}$ & $773 \pm 147$ & $\mathbf{1 0 2 8} \pm \mathbf{3 4}$ & $\mathbf{1 0 1 0} \pm \mathbf{6}$ & $794 \pm 49$ & $766 \pm 25$ & $1046 \pm 66$ \\
wino & $895 \pm 23$ & $\mathbf{7 4 8} \pm \mathbf{6 8}$ & $892 \pm 18$ & $958 \pm 10$ & $\mathbf{8 1 9} \pm \mathbf{4 7}$ & $911 \pm 51$ & $928 \pm 37$ \\
\hline \multicolumn{7}{|c|}{ scenario C } \\
\hline bino & $812 \pm 24$ & $545 \pm 8$ & $\mathbf{9 2 1} \pm \mathbf{3 7}$ & $816 \pm 29$ & $721 \pm 90$ & $708 \pm 22$ & $\mathbf{8 9 4} \pm \mathbf{5 7}$ \\
wino & $778 \pm 23$ & $577 \pm 19$ & $\mathbf{8 0 4} \pm \mathbf{6}$ & $769 \pm 47$ & $764 \pm 14$ & $708 \pm 38$ & $\mathbf{7 9 3} \pm \mathbf{7}$ \\
\hline
\end{tabular}

Table 2. Fit values for the discussed variables in the two endpoint selections, obtained with our own implementation of the edge-to-bump method [65]. The values closest to the true endpoints are highlighted in bold face.

sion is that no variable works perfectly for all scenarios, which suggests that one should carefully choose the variables depending on the mass spectrum and the endpoints (bino or wino) to be measured. ${ }^{4}$ In scenario $\mathrm{A}, m_{12}^{(2)}$ serves the best estimates of the bino and wino endpoints among all variables. $\min _{123}$ and $\min _{3 j}$ possess shifts of about $100 \mathrm{GeV}$ towards higher mass regions but preserve the correct difference between the wino and bino edges. In scenario $\mathrm{B}$, the $\tilde{q} \tilde{g}$ associated production gives an additional high $p_{T}$ jet and the size of the combinatorial background is the largest among the three scenarios. However, $\min _{123}, \min _{3 j}$ and $m_{12}^{(1)}\left(\min _{234}\right.$ and $\left.m_{12}^{(2)}\right)$ provide consistent results with the theoretically expected value for the bino (wino) edge measurement and the errors are somewhat smaller than in scenario A. On the other hand, the difference among the two endpoints are underestimated by most variables. This reflects the importance to use an appropriate variable depending on wether a wino or bino selection criterion is applied. In scenario C, all variables tend to underestimate the bino endpoint, despite the fact that the combinatorial and SUSY backgrounds are smallest in size among the three scenarios. This lower bias effect stems from poor statistics due to a small SUSY cross section in scenario C. ${ }^{5}$ We checked that the tendency of underestimation is removed when the number of events is increased artificially. For the wino edge, many variables, $\min _{123}, \min _{3 j}, m_{12}^{(1)}, F_{4}$, give good results with small errors.

Finally we want to stress that the quoted errors in table 2 are only errors originating from the fitting range dependence on fit results and there exist other sources of errors, which should be taken into account. For example, the statistical error on each bin content

\footnotetext{
${ }^{4} \mathrm{~A}$ rough idea of the type of mass spectra under investigation may for example be obtained from a jet number distribution (see figure 2), or the size of the signal cross section. In this study, however, we simply assume that such an observation has already been made at this stage of mass measurement.

${ }^{5}$ The linear kink fit method inevitably underestimates endpoints, if statistics are too low. This is simply because endpoints are not sufficiently populated in this limit. Such a situation strongly encourages a higher luminosity upgrade of the LHC (SLHC). Otherwise, more sophisticated approaches such as the template method may be required to correctly extract the gluino dijet endpoint(s).
} 
and the biases inherent in the according variable as well as backgrounds would all give contributions of the size of the errors we quoted. A careful estimation of these is beyond the scope of this paper but will be an important subject for gluino endpoint measurements.

\section{Conclusions}

In this work we studied the feasibility of the gluino dijet mass edge measurement in a realistic LHC environment. This includes both full SUSY backgrounds and combinatorial miscombinations of particles, as well as effects of initial and final state radiation and finite detector resolution. Several methods in the literature explicitly rely on the precise knowledge of a particular endpoint to be able to access information on masses in decay cascades, to resolve combinatorial issues, or determine another kinematical variable. Often QCD radiation and detector effects have been neglected in first phenomenological investigations. By utilizing considerations from the analysis of topological configurations of gluino decay cascades at the parton level, we find that the surviving correlation between the number of parton level and detector level jets is sufficient in distinguishing bino and wino endpoints of gluino decays with semi-inclusive jet multiplicities and the use of leptons as further selection criteria. To assess the impact of different mass spectra, we analyse three distinct (semi-)simplified models: two with small and large mass differences between gluino and squarks, and one scenario with decoupled scalars. In these models, we make use of existing kinematic variables and propose new ones, where necessary, that reduce the combinatorial problem and, when applied to preselected events, allow for the excavation of two distinct gluino endpoints. In general, the kinematic variables presented here are robust against contaminations from QCD radiation, underlying (non-signal) SUSY processes as well as detector effects. The resulting distributions are fitted with the so-called edge-to-bump method minimizing the artifact of the fit. These first estimates of the gluino dijet endpoints are mostly consistent with their expected values and thus prove the validity of our method. Hence, it seems possible to measure the gluino dijet edges for basically all cases where the squarks are heavier than the gluino, a parameter space that seems to be favored by recent Higgs search analyses from the LHC experiments.

\section{Acknowledgments}

We would like to thank Christian Sander and Matthias Schröder for valuable comments and helpful discussions. JRR and DW like to thank the Institute of Physics of the University of Freiburg for their hospitality.

Open Access. This article is distributed under the terms of the Creative Commons Attribution License which permits any use, distribution and reproduction in any medium, provided the original author(s) and source are credited.

\section{References}

[1] A.J. Barr and C.G. Lester, A review of the mass measurement techniques proposed for the Large Hadron Collider, J. Phys. G 37 (2010) 123001 [arXiv:1004.2732] [INSPIRE]. 
[2] I. Hinchliffe, F. Paige, M. Shapiro, J. Soderqvist and W. Yao, Precision SUSY measurements at CERN LHC, Phys. Rev. D 55 (1997) 5520 [hep-ph/9610544] [INSPIRE].

[3] H. Baer, C.-h. Chen, M. Drees, F. Paige and X. Tata, Probing minimal supergravity at the CERN LHC for large tan $\beta$, Phys. Rev. D 59 (1999) 055014 [hep-ph/9809223] [INSPIRE].

[4] I. Hinchliffe and F. Paige, Measurements in gauge mediated SUSY breaking models at CERN LHC, Phys. Rev. D 60 (1999) 095002 [hep-ph/9812233] [inSPIRE].

[5] H. Bachacou, I. Hinchliffe and F.E. Paige, Measurements of masses in SUGRA models at CERN LHC, Phys. Rev. D 62 (2000) 015009 [hep-ph/9907518] [INSPIRE].

[6] I. Hinchliffe and F. Paige, Measurements in SUGRA models with large $\tan \beta$ at CERN LHC, Phys. Rev. D 61 (2000) 095011 [hep-ph/9907519] [INSPIRE].

[7] B. Allanach, C. Lester, M.A. Parker and B. Webber, Measuring sparticle masses in nonuniversal string inspired models at the LHC, JHEP 09 (2000) 004 [hep-ph/0007009] [INSPIRE].

[8] C. Lester and D. Summers, Measuring masses of semiinvisibly decaying particles pair produced at hadron colliders, Phys. Lett. B 463 (1999) 99 [hep-ph/9906349] [INSPIRE].

[9] A. Barr, C. Lester and P. Stephens, $M_{T 2}$ : the truth behind the glamour, J. Phys. G G 29 (2003) 2343 [hep-ph/0304226] [INSPIRE].

[10] W.S. Cho, K. Choi, Y.G. Kim and C.B. Park, Gluino stransverse mass, Phys. Rev. Lett. 100 (2008) 171801 [arXiv:0709.0288] [INSPIRE].

[11] W.S. Cho, K. Choi, Y.G. Kim and C.B. Park, Measuring superparticle masses at hadron collider using the transverse mass kink, JHEP 02 (2008) 035 [arXiv:0711.4526] [INSPIRE].

[12] B. Gripaios, Transverse observables and mass determination at hadron colliders, JHEP 02 (2008) 053 [arXiv:0709.2740] [InSPIRE].

[13] A.J. Barr, B. Gripaios and C.G. Lester, Weighing wimps with kinks at colliders: invisible particle mass measurements from endpoints, JHEP 02 (2008) 014 [arXiv:0711.4008] [INSPIRE].

[14] M. Burns, K. Kong, K.T. Matchev and M. Park, Using subsystem MT2 for complete mass determinations in decay chains with missing energy at hadron colliders, JHEP 03 (2009) 143 [arXiv:0810.5576] [INSPIRE].

[15] P. Konar, K. Kong, K.T. Matchev and M. Park, Superpartner mass measurement technique using $1 D$ orthogonal decompositions of the Cambridge transverse mass variable $M_{T 2}$, Phys. Rev. Lett. 105 (2010) 051802 [arXiv:0910.3679] [INSPIRE].

[16] T. Cohen, E. Kuflik and K.M. Zurek, Extracting the dark matter mass from single stage cascade decays at the LHC, JHEP 11 (2010) 008 [arXiv: 1003.2204] [INSPIRE].

[17] A.J. Barr, B. Gripaios and C.G. Lester, Transverse masses and kinematic constraints: from the boundary to the crease, JHEP 11 (2009) 096 [arXiv:0908.3779] [INSPIRE].

[18] P. Konar, K. Kong, K.T. Matchev and M. Park, Dark matter particle spectroscopy at the LHC: generalizing $M_{T 2}$ to asymmetric event topologies, JHEP 04 (2010) 086 [arXiv: 0911.4126] [INSPIRE].

[19] C. Lester and A. Barr, MTGEN: mass scale measurements in pair-production at colliders, JHEP 12 (2007) 102 [arXiv:0708.1028] [INSPIRE]. 
[20] M.M. Nojiri, Y. Shimizu, S. Okada and K. Kawagoe, Inclusive transverse mass analysis for squark and gluino mass determination, JHEP 06 (2008) 035 [arXiv:0802.2412] [INSPIRE].

[21] M.M. Nojiri, K. Sakurai, Y. Shimizu and M. Takeuchi, Handling jets + missing $E_{T}$ channel using inclusive $M_{T 2}$, JHEP 10 (2008) 100 [arXiv:0808.1094] [INSPIRE].

[22] S.-G. Kim, N. Maekawa, K.I. Nagao, M.M. Nojiri and K. Sakurai, LHC signature of supersymmetric models with non-universal sfermion masses, JHEP 10 (2009) 005 [arXiv: 0907.4234] [INSPIRE].

[23] D.R. Tovey, On measuring the masses of pair-produced semi-invisibly decaying particles at hadron colliders, JHEP 04 (2008) 034 [arXiv:0802.2879] [INSPIRE].

[24] G. Polesello and D.R. Tovey, Supersymmetric particle mass measurement with the boost-corrected contransverse mass, JHEP 03 (2010) 030 [arXiv:0910.0174] [INSPIRE].

[25] G.G. Ross and M. Serna, Mass determination of new states at hadron colliders, Phys. Lett. B 665 (2008) 212 [arXiv:0712.0943] [INSPIRE].

[26] A. Barr et al., Guide to transverse projections and mass-constraining variables, Phys. Rev. D 84 (2011) 095031 [arXiv:1105.2977] [INSPIRE].

[27] B. Gripaios, A. Papaefstathiou, K. Sakurai and B. Webber, Searching for third-generation composite leptoquarks at the LHC, JHEP 01 (2011) 156 [arXiv:1010.3962] [INSPIRE].

[28] A.J. Barr, S.T. French, J.A. Frost and C.G. Lester, Speedy Higgs boson discovery in decays to tau lepton pairs : $h \rightarrow \tau, \tau, J H E P 10$ (2011) 080 [arXiv:1106.2322] [INSPIRE].

[29] L. Harland-Lang, C. Kom, K. Sakurai and W. Stirling, Measuring the masses of a pair of semi-invisibly decaying particles in central exclusive production with forward proton tagging, Eur. Phys. J. C 72 (2012) 1969 [arXiv:1110.4320] [INSPIRE].

[30] L. Harland-Lang, C. Kom, K. Sakurai and W. Stirling, Mass shell technique for measuring masses of a pair of semi-invisibly decaying particles, Phys. Rev. Lett. 108 (2012) 181805 [arXiv: 1202.0047] [INSPIRE].

[31] M. Serna, A short comparison between $M_{T 2}$ and $m_{C T}$, JHEP 06 (2008) 004 [arXiv:0804.3344] [INSPIRE].

[32] H.-C. Cheng and Z. Han, Minimal kinematic constraints and $M_{T 2}$, JHEP 12 (2008) 063 [arXiv: 0810.5178] [INSPIRE].

[33] K. Desch, J. Kalinowski, G.A. Moortgat-Pick, M. Nojiri and G. Polesello, SUSY parameter determination in combined analyses at LHC/LC, JHEP 02 (2004) 035 [hep-ph/0312069] [INSPIRE].

[34] K. Kawagoe, M. Nojiri and G. Polesello, A New SUSY mass reconstruction method at the CERN LHC, Phys. Rev. D 71 (2005) 035008 [hep-ph/0410160] [INSPIRE].

[35] H.-C. Cheng, J.F. Gunion, Z. Han, G. Marandella and B. McElrath, Mass determination in SUSY-like events with missing energy, JHEP 12 (2007) 076 [arXiv:0707.0030] [INSPIRE].

[36] H.-C. Cheng, D. Engelhardt, J.F. Gunion, Z. Han and B. McElrath, Accurate mass determinations in decay chains with missing energy, Phys. Rev. Lett. 100 (2008) 252001 [arXiv: 0802.4290] [INSPIRE].

[37] H.-C. Cheng, J.F. Gunion, Z. Han and B. McElrath, Accurate mass determinations in decay chains with missing energy. II, Phys. Rev. D 80 (2009) 035020 [arXiv:0905.1344] [INSPIRE]. 
[38] B. Webber, Mass determination in sequential particle decay chains, JHEP 09 (2009) 124 [arXiv:0907.5307] [INSPIRE].

[39] M.M. Nojiri, K. Sakurai and B.R. Webber, Reconstructing particle masses from pairs of decay chains, JHEP 06 (2010) 069 [arXiv:1005.2532] [INSPIRE].

[40] P. Konar, K. Kong and K.T. Matchev, $\sqrt{\hat{s}}_{\text {min }}$ : a global inclusive variable for determining the mass scale of new physics in events with missing energy at hadron colliders, JHEP 03 (2009) 085 [arXiv:0812.1042] [INSPIRE].

[41] P. Konar, K. Kong, K.T. Matchev and M. Park, RECO level $\sqrt{s}_{\min }$ and subsystem $\sqrt{s}_{\min }$ : improved global inclusive variables for measuring the new physics mass scale in $E_{T}$ events at hadron colliders, JHEP 06 (2011) 041 [arXiv: 1006.0653] [INSPIRE].

[42] W.S. Cho, D. Kim, K.T. Matchev and M. Park, Cracking the dark matter code at the LHC, arXiv:1206.1546 [INSPIRE].

[43] Y. Bai and H.-C. Cheng, Identifying dark matter event topologies at the LHC, JHEP 06 (2011) 021 [arXiv: 1012.1863] [INSPIRE].

[44] B. Gripaios, K. Sakurai and B. Webber, Polynomials, Riemann surfaces and reconstructing missing-energy events, JHEP 09 (2011) 140 [arXiv:1103.3438] [INSPIRE].

[45] B. Dutta, T. Kamon, N. Kolev and A. Krislock, Bi-event subtraction technique at hadron colliders, Phys. Lett. B 703 (2011) 475 [arXiv:1104.2508] [inSPIRE].

[46] A. Rajaraman and F. Yu, A new method for resolving combinatorial ambiguities at hadron colliders, Phys. Lett. B 700 (2011) 126 [arXiv:1009.2751] [INSPIRE].

[47] P. Baringer, K. Kong, M. McCaskey and D. Noonan, Revisiting combinatorial ambiguities at hadron colliders with $M_{T 2}$, JHEP 10 (2011) 101 [arXiv:1109.1563] [INSPIRE].

[48] K. Choi, D. Guadagnoli and C.B. Park, Reducing combinatorial uncertainties: a new technique based on $M_{T 2}$ variables, JHEP 11 (2011) 117 [arXiv:1109.2201] [inSPIRE].

[49] J. Alwall, K. Hiramatsu, M.M. Nojiri and Y. Shimizu, Novel reconstruction technique for New Physics processes with initial state radiation, Phys. Rev. Lett. 103 (2009) 151802 [arXiv:0905.1201] [INSPIRE].

[50] M.M. Nojiri and K. Sakurai, Controlling ISR in sparticle mass reconstruction, Phys. Rev. D 82 (2010) 115026 [arXiv:1008.1813] [INSPIRE].

[51] K. Hagiwara et al., Supersymmetry simulations with off-shell effects for CERN LHC and ILC, Phys. Rev. D 73 (2006) 055005 [hep-ph/0512260] [INSPIRE].

[52] CMS collaboration, G. Bayatian et al., CMS technical design report, volume II: physics performance, J. Phys. G 34 (2007) 995 [inSPIRE].

[53] M. Bahr et al., HERWIG++ physics and manual, Eur. Phys. J. C 58 (2008) 639 [arXiv: 0803.0883] [INSPIRE].

[54] W. Kilian, T. Ohl and J. Reuter, WHIZARD: simulating multi-particle processes at LHC and ILC, Eur. Phys. J. C 71 (2011) 1742 [arXiv:0708.4233] [INSPIRE].

[55] M. Moretti, T. Ohl and J. Reuter, O'Mega: an optimizing matrix element generator, hep-ph/0102195 [INSPIRE].

[56] W. Kilian, J. Reuter, S. Schmidt and D. Wiesler, An analytic initial-state parton shower, JHEP 04 (2012) 013 [arXiv:1112.1039] [INSPIRE]. 
[57] W. Beenakker, R. Hopker, M. Spira and P. Zerwas, Squark and gluino production at hadron colliders, Nucl. Phys. B 492 (1997) 51 [hep-ph/9610490] [INSPIRE].

[58] S. Ovyn, X. Rouby and V. Lemaitre, DELPHES, a framework for fast simulation of a generic collider experiment, arXiv:0903.2225 [INSPIRE].

[59] M. Cacciari, G.P. Salam and G. Soyez, The anti- $k_{t}$ jet clustering algorithm, JHEP 04 (2008) 063 [arXiv:0802.1189] [INSPIRE].

[60] M. Cacciari, G.P. Salam and G. Soyez, FastJet user manual, Eur. Phys. J. C 72 (2012) 1896 [arXiv: 1111.6097] [INSPIRE].

[61] M. Cacciari and G.P. Salam, Dispelling the $N^{3}$ myth for the $k_{t}$ jet-finder, Phys. Lett. B 641 (2006) 57 [hep-ph/0512210] [INSPIRE].

[62] CMS collaboration, S. Chatrchyan et al., Search for new physics with jets and missing transverse momentum in pp collisions at $\sqrt{s}=7 \mathrm{TeV}$, JHEP 08 (2011) 155 [arXiv:1106.4503] [INSPIRE].

[63] ATLAS collaboration, G. Aad et al., Search for new phenomena in final states with large jet multiplicities and missing transverse momentum using $\sqrt{s}=7 \mathrm{TeV}$ pp collisions with the ATLAS detector, JHEP 11 (2011) 099 [arXiv:1110.2299] [INSPIRE].

[64] CMS collaboration, S. Chatrchyan et al., Search for supersymmetry in pp collisions at $\sqrt{s}=7 \mathrm{TeV}$ in events with a single lepton, jets and missing transverse momentum, JHEP 08 (2011) 156 [arXiv:1107.1870] [INSPIRE].

[65] D. Curtin, Mixing it up with $M_{T 2}$ : unbiased mass measurements at hadron colliders, Phys. Rev. D 85 (2012) 075004 [arXiv:1112.1095] [INSPIRE]. 